\title{
Verkürzter Penis nach Prostatektomie?
}

Gelegentlich klagen Männer nach einer Prostatektomie über einen verkürzten Penis. Ob dies von der Art der Therapie abhängt und mit der Therapiezufriedenheit korreliert, haben US-amerikanische Forscher untersucht.

elegentlich haben Männer mit Prostatakarzinom den Eindruck, dass nach der Therapie ihr Penis verkürzt ist. Wie häufig das bei einzelnen Therapien ist, haben nun US-amerikanische Onkologen bei 948 Männern mit einem PSARezidiv eines Prostatakarzinoms ermittelt. Zu den abgefragten Komplikationen zählte auch ein verkürzter Penis. Insge- samt hatten $25(2,6 \%)$ der Teilnehmer nach der Therapie den Eindruck, ihr Penis sei verkürzt, am häufigsten nach Prostatektomie: Hier waren es 19 von 510 (3,7\%) Männern, nach Androgendeprivation plus Radiotherapie sechs von 225 $(2,7 \%)$ und nach alleiniger Radiotherapie keiner von 213 Patienten (Abb.). Trotz der geringen Zahlen waren die Unterschiede

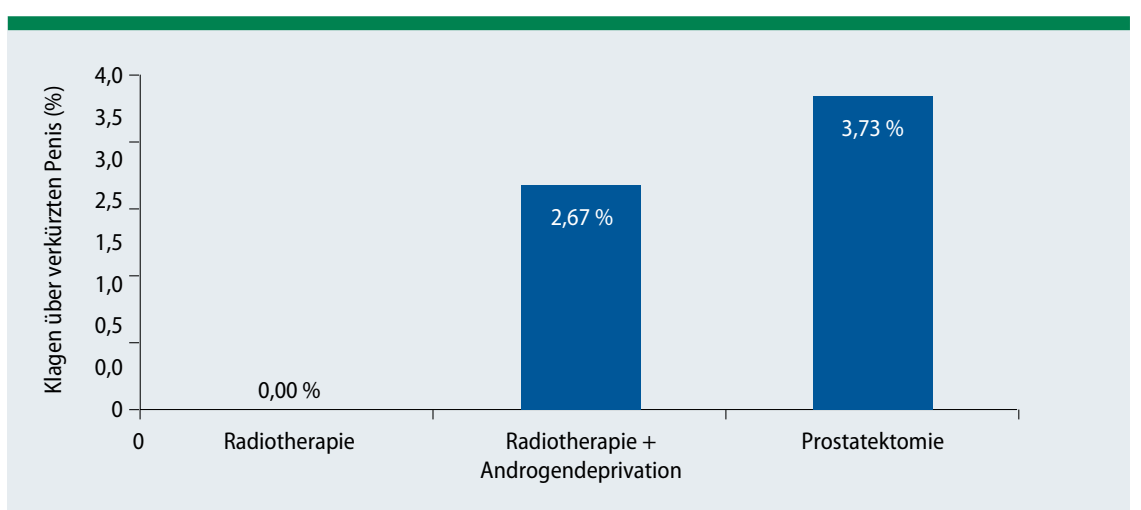

Abb.: Anteil der Patienten in den einzelnen Therapiegruppen, die angeben, ihr Penis habe sich verkürzt.

\section{Prostatakarzinom: Akzeptable Toxizität bei $86,4 \mathrm{~Gy}$}

\begin{abstract}
Mit intensitätsmodulierter Radiotherapie (IMRT) lassen sich Dosen von mehr als 86,4 Gy in die Prostata applizieren. Über Outcome und Toxizität bei mehr als 1.000 Patienten mit lokalem Prostatakarzinom liegen jetzt Langzeitdaten vor.
\end{abstract}

S chon bald, nachdem die External $S$ Beam Radiation Therapy (EBRT) in die Therapie des Prostatakarzinoms implementiert wurde, zeigte sich: Mit dieser Methode ließen sich erheblich bessere Ergebnisse erzielen, wenn Strahlendosen von mehr als 70 Gy verabreicht werden könnten. Nach und nach wurden seither mittels IMRT immer höhere Dosen verabreicht - Kollegen vom Memorial Sloan-Kettering Cancer Center gehen beim lokalisierten Prostatakarzinom inzwischen auf 86,4 Gy. Sie präsentieren ein Langzeit-Follow-up für 1.002
Patienten, die zwischen 1997 und 2008 mit einer 5-7-Feld-IMRT behandelt worden waren. Sie wurden in drei Risikogruppen eingeteilt. Das mediane Followup lag bei 5,5 Jahren. Die Ergebnisse für die drei Gruppen mit niedrigem, mittlerem bzw. hohem Risiko waren wie folgt: -Frei von biochem. Rezidiv nach 7 Jahren: $98,8 \%, 85,6 \%$ bzw. $67,9 \%$.

_Frei von Fernmetastasen: 99,4\%, $94,1 \%$ bzw. $82,0 \%$.

_Prostatakarzinom-spezifische 7-Jahres-Mortalität: $0 \%, 3,3 \%$ bzw. 8,1\%. -Gastrointestinale oder urogenitale zwischen der letzten und den anderen beiden Gruppen signifikant. Auffallend war zudem, dass Männer, die einen verkürzten Penis angaben, häufiger als andere ihre Entscheidung bereuten und auch sexuelle Probleme hatten. Allerdings war die Therapiezufriedenheit in der Prostatektomie-Gruppe insgesamt am höchsten: Hier bereute ein Drittel weniger die Wahl als bei alleiniger Radiotherapie.

Die Männer bilden sich die Penisverkürzung nach einer Op. nicht nur ein: In einer anderen Studie war das männliche Genitale ein Monat nach nervenerhaltender Prostatektomie im Schnitt über einen halben Zentimeter kürzer, was mit einer postoperativen Degeneration peniler Nervenfasern erklärt wird.

Fazit: Männer sollten vor einer Prostatektomie oder Androgendeprivation darüber aufgeklärt werden, dass diese ihren Penis verkürzen kann, wenngleich dies nur selten als Problem empfunden wird. Mit diesem Wissen kann spätere Unzufriedenheit mit dem Therapieergebnis eher vermieden werden. Thomas Müller

Parekh A et al. Reduced penile size and treatment regret in men with recurrent prostate cancer after surgery, radiotherapy plus androgen deprivation, or radiotherapy alone. Urology. 2013;81(1):130-4.

Spätfolgen vom Grad 2 oder mehr: $0 \%, 4,4 \%$ bzw. $21,1 \%$.

_Gastrointestinale oder urogenitale Spät- folgen vom Grad 3: $0 \%, 0,7 \%$ bzw. $2,2 \%$.

$\mathrm{Zu}$ Beginn der Behandlung litten 427 Männer nicht unter sexueller Dysfunktion. Zum Zeitpunkt der letzten Untersuchung wurde eine solche bei 110 Männern (26\%) festgestellt.

Fazit: Die intensive Bestrahlung mit 86,4 Gy führte in dieser Studie zu sehr guten klinischen Resultaten bei akzeptabler Toxizität. Es ist aber nötig, sich bei diesen hohen Dosen besonders strikt an die Protokolle zu halten.

Christina Berndt

Spratt DE et al. Long-term survival and toxicity in patients treated with high-dose intensity modulated radiation therapy for localized prostate cancer. Intern J Rad Oncol Biol Phys. 2013; 85(3):686-92. 